\title{
A molecular dynamics study on melting point and specific heat of $\mathrm{Ni}_{3} \mathrm{Al}$ alloy
}

\author{
YANG Hong, LÜ YongJun, CHEN Min ${ }^{\dagger} \&$ GUO ZengYuan \\ Department of Engineering Mechanics, Tsinghua University, Beijing 100084, China
}

Using the Embedding Atom Method (EAM) for highly undercooled $\mathrm{Ni}_{3} \mathrm{Al}$ alloy, the melting point and the specific heat were studied by a molecular dynamics simulation. The simulation of melting point was carried out by means of the sandwich method and the NVE ensemble method, and the results show a good agreement, whereas are larger than the experimental value of $1663 \mathrm{~K}$. This difference is attributed to the influence of surface melting on experimental results, which causes the smaller measurements compared with the thermodynamic melting point. The simulated specific heat of $\mathrm{Ni}_{3} \mathrm{Al}$ alloy weakly and linearly increases with the increase of undercooling in the temperature range from $800 \mathrm{~K}$ to $2000 \mathrm{~K}$.

$\mathrm{Ni}_{3} \mathrm{Al}$ alloy, undercooled, melting point, specific heat

The rapid solidification process with low cooling rate can be obtained by using high undercooling conditions, which overcomes the limit of heat transfer during the quenching and is an effective manner to carry out the rapid solidification of bulk melts. The quantitative investigation of the rapid solidification under high undercooling requires enough accurate thermodynamic data of undercooled melts, whereas they are often lacking in handbook ${ }^{[1]}$. To date, the experimental measurements of thermodynamic properties of undercooled metal are performed by various levitation technologies such as electromagnetic levitation and acoustic levitation ${ }^{[2,3]}$. These containerless processing methods can effectively avoid the heterogeneous nucleation induced by the container wall and are in favor of large undercooling. Due to the high cost of experimental measurements, however, these studies focus on a few metal and alloy systems, and correspondingly the thermodynamic data are limited. The molecular dynamics simulation can easily produce the underooling state of metal and a potential method to study the thermodynamic properties of metastable metals, and permits to further detect the intrinsic correlation between the thermodynamic property and the microstructure at a molecular level ${ }^{[4,5]}$.

As a basic thermodynamic parameter, the melting point is beneficial for revealing the phase choice during the solidification and determining the undercooling. The specific heat plays an im-

Received February 11, 2007; accepted April 16, 2007 doi: $10.1007 / \mathrm{s} 11433-007-0039-5$

†Corresponding author (email: mchen@tsinghua.edu.cn)

Supported by the National Natural Science Foundation of China (Grant No. 50395101) 
portant role in non-equilibrium liquid/solid transition. Given the specific heat under a certain undercoooling, the latent heat, entropy change and free energy change during crystallization are available, which are necessary for studying nucleation and crystal growth of undercooled melts. As a kind of high-temperature structural material, $\mathrm{Ni}_{3} \mathrm{Al}$ alloy is characterized by the high melting point and excellent oxidation resistance and has a wide application in the aero-industry. In this paper, the melting point and the temperature dependent specific heat of $\mathrm{Ni}_{3} \mathrm{Al}$ alloy are studied by using the molecular dynamics method.

\section{Potential function}

The interaction between $\mathrm{Ni}$ and $\mathrm{Al}$ atoms is described by the EAM potential function, in which every atom is embedded in the matrix of other atoms. The total energy is written as the sum of embedding energy and pair potential, and given in the following form ${ }^{[6]}$ :

$$
\begin{gathered}
E_{i}=F_{i}\left(\rho_{h, i}\right)+\sum_{i} \sum_{j} \varphi_{i j}\left(r_{i j}\right), \\
\rho_{h, i}=\sum_{j \neq i} f_{j}\left(r_{i j}\right),
\end{gathered}
$$

where $E_{i}$ is the energy for atom $i, \rho_{h, i}$ the background electron density for atom $i, f_{i}$ the electron density contribution of atom $j$ to $i, r_{i, j}$ the distance between atoms $i$ and $j, F_{i}\left(\rho_{h, i}\right)$ the embedding function for atom $i$ embedded in a background electron density, and $\varphi_{i j}\left(r_{i j}\right)$ the pair potential between atoms $i$ and $j$.

Voter and Chen ${ }^{[7]}$ developed the EAM potential applicable to $\mathrm{Ni}$-Al alloy system according to $\mathrm{Ni}_{3} \mathrm{Al}$ alloy, i.e. $\mathrm{VC}$ potential, and the pair potential is given by

$$
\varphi(r)=D_{\mathrm{M}}\left\{1-\exp \left[-\alpha_{\mathrm{M}}\left(r-R_{\mathrm{M}}\right)\right]\right\}^{2}-D_{\mathrm{M}} .
$$

The background electron density is given in the following form:

$$
f(r)=r^{6}\left(\mathrm{e}^{-\beta r}+2^{9} \mathrm{e}^{-2 \beta r}\right) .
$$

In the present simulation, the pair potential and the background electron density are smoothed as follows:

$$
A_{\text {smooth }}=A(r)-A\left(r_{\text {cut }}\right)+\left(\frac{r_{\text {cut }}}{m}\right)\left[1-\left(\frac{r}{r_{\text {cut }}}\right)^{m}\right]\left(\frac{\mathrm{d} A}{\mathrm{~d} r}\right)_{r=r_{\text {cut }}},
$$

where $A(r)$ can be $\varphi(r)$ and $\rho(r)$, respectively, and $r_{\text {cut }}$ is cutoff radius. In calculations, the parameters used in eqs. (3)-(5) are given in Table 1. The embedding energy in VC potential can be obtained by fitting the Rose equation that describes the energy for the defective crystal. Combining the Rose Equation, pair potential, background electron density and eq. (1), we can get the embedding energy.

Table 1 Model parameters used in the pair potential and the background electron density

\begin{tabular}{cccccc}
\hline & $D_{\mathrm{M}}(\mathrm{eV})$ & $R_{\mathrm{M}}(\AA)$ & $\alpha_{\mathrm{M}}\left(\AA^{-1}\right)$ & $\beta\left(\AA^{-1}\right)$ & 3.6408 \\
$\mathrm{Ni}$ & 1.5335 & 2.2053 & 1.7728 & 3.3232 & 5.7895 \\
$\mathrm{Al}$ & 3.7760 & 2.1176 & 1.4859 & -5.5550 & 5.4639 \\
$\mathrm{Ni}-\mathrm{Al}$ & 3.0322 & 2.0896 & 1.6277 & - & 5 \\
\hline
\end{tabular}




\section{Melting point}

The thermodynamic melting point is the temperature $T_{\mathrm{m}}$, at which the chemical potentials of liquid and solid phases are identical and the coexistence between them is stable. Due to the interfacial energy, the calculated melting point by directly heating the large simulation system usually is larger than the thermodynamic melting point. Therefore, the liquid/solid interface should be constructed properly. There are three methods to simulate the melting point. The first one is the sandwich method. We constructed a "solid-liquid-solid" interlayer structure firstly, and then observed the evolution of such a metastable structure during annealing ${ }^{[8-10]}$. If the sandwich structure completely develops into the liquid state, the current temperature is higher than the melting point, and, contrariwise, lower than the melting point. If the liquid/solid structure can stably exist, the temperature is the melting point. The second method is the moving interface method ${ }^{[11]}$. Firstly, we constructed a liquid/solid interface, and then measured the moving velocity of the interface at different temperatures. After that, we extrapolated the moving velocity to zero, and the corresponding temperature was the melting point. The third one is the NVE ensemble method. Similar to the above method, the liquid/solid double-layer structure was set up firstly. Then, the structure evolved in NVE ensemble and the final equilibrium temperature was the melting point ${ }^{[12,13]}$.

In this work, the melting point is simulated by employing the sandwich and NVE ensemble methods, respectively.

\subsection{Sandwich method}

The "solid-liquid-solid" sandwich structure was constructed firstly. The simulation cell was a rectangular with $750 \mathrm{Ni}$ atoms and $250 \mathrm{Al}$ atoms. Periodic boundary conditions were applied in the three coordinate directions, and the "solid-liquid-solid" multi-layer was arranged in the $z$ direction. A liquid layer with 500 atoms was located in the middle and two solid layers in each side of the cell. At the beginning of the simulation, the two solid layers and the liquid layer were equilibrated at two different temperatures, respectively. The solid temperature was lower than the melting point and the liquid temperature was larger than that. After the equilibrium was reached for the three sub-systems, the three layers were integrated into a sandwich system. The system was equilibrated in an ensemble at a constant temperature. At a series of trial temperature, we can plot the system energy as a function of temperature, which helps us to determine the melting point easily.

Figure 1 shows the energy versus temperature by the sandwich structure method. It can be seen that there exists a singularity between 1700 and $1710 \mathrm{~K}$, and the system energy shows an abrupt change. Before and after this abrupt change, the energy is characterized by the liquid and the solid states. Therefore, the temperature corresponding to the singularity, $T^{*} \approx(1705 \pm 5) \mathrm{K}$, is the melting point.

\subsection{NVE ensemble method}

The solid and liquid systems with the same volume and atom number were equilibrated near the melting point in an NVT ensemble, and their equilibrium configurations were recorded. Then the initial solid and liquid configurations were jointed together to form a new system, which was equilibrated in an NVE ensemble. After the equilibrium, the temperature was the melting point ${ }^{[14,15]}$. In fact, if the initial temperature is larger than the melting point, the solid phase will melt and the equilibrium temperature will be lowered due to the energy conservation. Contrarily, 


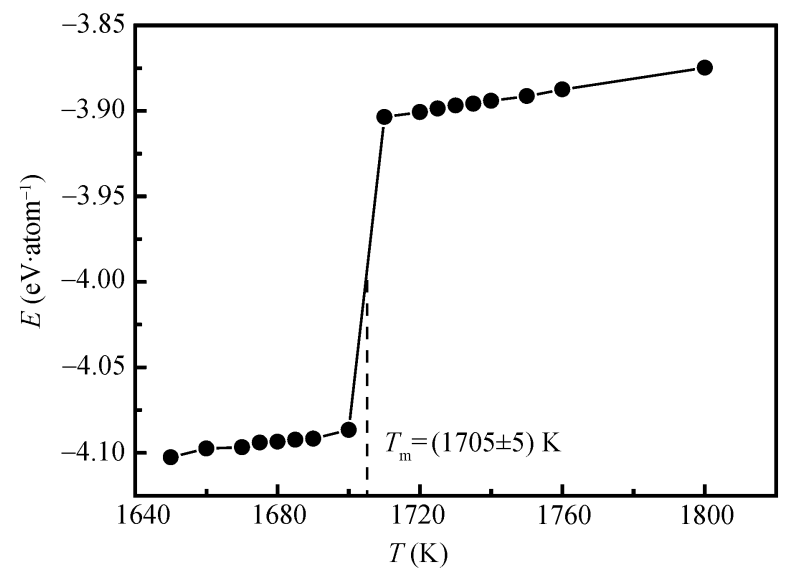

Figure 1 Internal energy of $\mathrm{Ni}_{3} \mathrm{Al}$ alloy versus temperature.

the equilibrium temperature will be raised by the release of latent during the liquid crystallization provided the initial temperature is smaller than the melting point. Therefore, the simulated system will be stabilized at the melting point. In order to accelerate the equilibrium process, the initial temperature should be set closely near the melting point.

Figure 2 shows the kinetic energy as a function of equilibrium steps. With the proceeding of equilibrating, the system kinetic energy approaches a constant. According to the energy asymptotic value, the melting point of $\mathrm{Ni}_{3} \mathrm{Al}$ alloy is determined as $1727.8 \mathrm{~K}$.

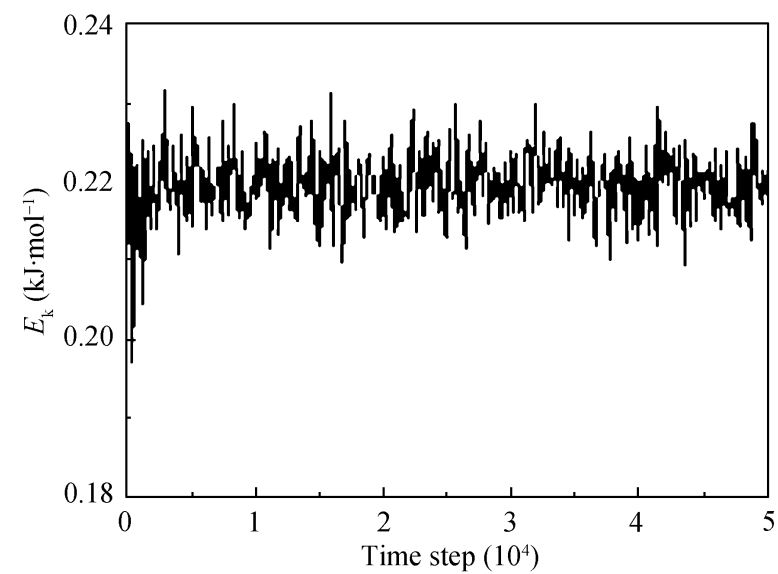

Figure 2 Kinetic energy evolves with the equilibrium step in an NVE ensemble.

\subsection{Comparison with the experiments}

Table 2 shows the comparison between the calculated melting point and the experimental value ${ }^{[16]}$. The two simulated values are in agreement by using the sandwich and the NVE method, and moreover they agree well with the experimental value. On the other hand, both the simulated values are larger than the experimental value by $2.5 \%$ and $3.8 \%$. Although the molecular dynamics simulations introduce some error, the behavior that both the simulated results are larger than the experimental results suggests an intrinsic factor. In experiments, the measurements of melting 
point are often influenced by the surface melting ${ }^{[17]}$. The melting process of metal initiates from the surface pre-melting, and the temperature corresponding to the surface melting is usually larger than the thermodynamic melting point, which accounts for the present larger simulated results.

Table 2 Comparison between the calculated melting point and the experimental value

\begin{tabular}{cccc}
\hline & Simulations $(\mathrm{K})$ & Experiments $(\mathrm{K})$ & Error \\
\hline Sandwich method & 1705 & 1663 & $2.5 \%$ \\
NVE method & 1727 & 1663 & $3.8 \%$ \\
\hline
\end{tabular}

\section{Specific heat}

The NPT molecular dynamics simulation was performed using the system of $1000 \mathrm{Ni}$ and Fe atoms with a time step of $10^{-15} \mathrm{~s}$. Periodic boundary conditions were applied in three coordinate directions. The initial configuration consisting of $250 \mathrm{Al}$ atoms and $750 \mathrm{Ni}$ atoms were arranged into body-centred cubic (bcc) lattice. The system with the initial temperature of $2000 \mathrm{~K}$ was quenched and sampled every $200 \mathrm{~K}$. The system was equilibrated for $10^{5}$ steps and the production ran for $5 \times 10^{4}$ steps. The minimum quenching temperature reached $800 \mathrm{~K}$ and the corresponding undercooling was up to $900 \mathrm{~K}$.

Two methods were applied to calculating the specific heat. The first one was carried out by means of the statistic of energy fluctuation, which often requires the system characterized by a large amount of atoms and relatively simple interactions. The second method is the derivative of the thermodynamic function:

$$
C_{\mathrm{PL}}=\frac{\mathrm{d} h}{\mathrm{~d} T} \text {. }
$$

The method is suitable for a small system and also shows a good precision, which is the simulation method used in the present work.

The calculated enthalpy of undercooled $\mathrm{Ni}_{3} \mathrm{Al}$ alloy as a function of temperature is shown in Figure 3. The enthalpy increases with the increase of temperature as follows:

$$
h(T)=-4.43 \times 10^{5}+38.7 T-0.00336 T^{2} \quad\left(\mathrm{~J} \cdot \mathrm{mol}^{-1}\right) .
$$

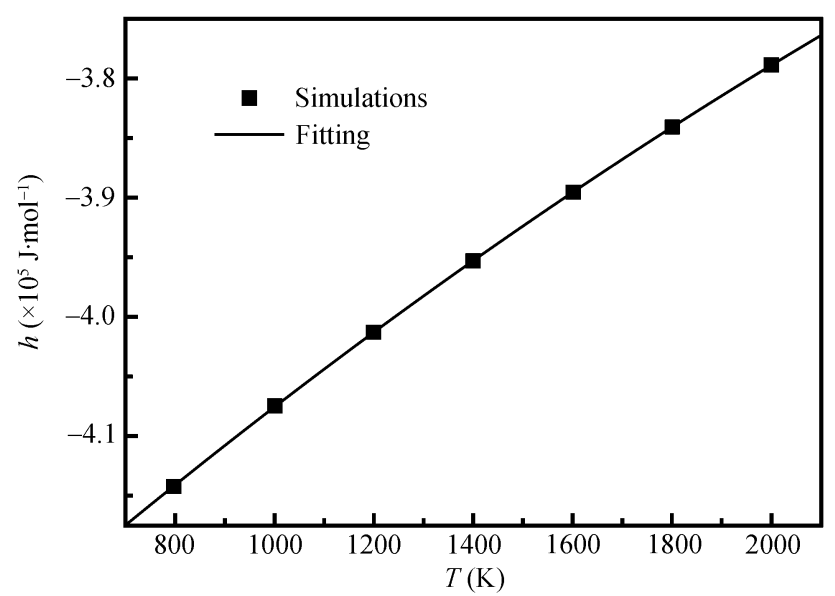

Figure 3 Enthalpy for $\mathrm{Ni}_{3} \mathrm{Al}$ alloy as a function of temperature. 
By the partial derivative of the above enthalpy to temperature, the specific heat as a function of temperature between 800 and $2000 \mathrm{~K}$ is shown in Figure 4. Similar to the temperature dependent specific heat of many alloy systems, the specific heat of $\mathrm{Ni}_{3} \mathrm{Al}$ alloy slowly and linearly increases as the temperature decreases:

$$
C_{\mathrm{PL}}=38.7-0.00672 T \quad\left(\mathrm{~J} \cdot \mathrm{mol}^{-1} \cdot \mathrm{K}^{-1}\right) .
$$

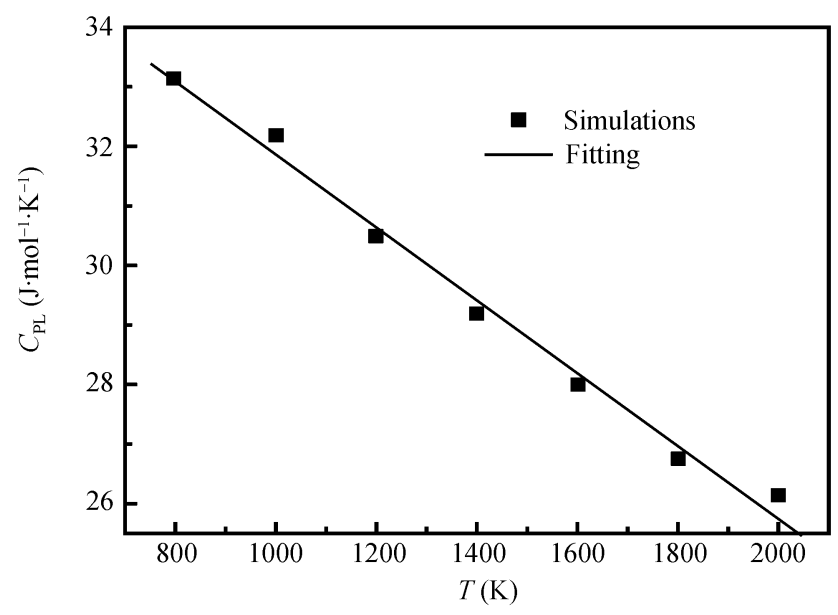

Figure 4 Specific heat of undercooled $\mathrm{Ni}_{3} \mathrm{Al}$ alloy versus temperature.

The specific heat varies within the range of $29.64 \pm 3.50 \mathrm{~J} \cdot \mathrm{mol}^{-1} \cdot \mathrm{K}^{-1}$ distributed among a wide temperature range of $1200 \mathrm{~K}$, indicating a weak temperature dependence of specific heat. Up to now, few experimental results about undercooled $\mathrm{Ni}_{3} \mathrm{Al}$ alloy have been reported, and the present results only provide a prediction for further investigations.

\section{Conclusions}

(1) The melting point of $\mathrm{Ni}_{3} \mathrm{Al}$ alloy was simulated by using the sandwich method and NVE ensemble method, and the results show $2.5 \%$ and $3.8 \%$ larger than the experimental value, which is attributed to the influence of the surface melting.

(2) The specific heat of undercooled $\mathrm{Ni}_{3} \mathrm{Al}$ alloy as a function of temperature was calculated, and the values show a linear temperature dependence,

$$
C_{\mathrm{PL}}=38.7-0.00672 T \quad\left(\mathrm{~J} \cdot \mathrm{mol}^{-1} \cdot \mathrm{K}^{-1}\right) .
$$

1 Wei B B, Tang G C, Zhou Y H. Solidification of highly undercooled metallic melts. Acta Aeronautica Et Astronautica Sinica (in Chinese), 1991, 12(5): A213-A220

2 Dai F P, Cao C D, Wei B B. Thermophysical properties of Ni-5\% Sn alloy melt. Sci China Ser G-Phys Mech Astron, 2006, 49(2): $236-245$

3 Wang H P, Wei B B. Surface tension and specific heat of liquid $\mathrm{M}_{70.2} \mathrm{Si}_{29.8}$ alloy. Chin Sci Bull, 2005, 50(10): 945 - 949

4 Han X J, Chen M, Guo Z Y. Thermophysical properties of undercooled liquid Au-Cu alloys by a molecular dynamics simulation. J Phys-Condens Matter, 2004, 16(6): 705-713

5 Wang J Z, Yang C, Chen M, et al. Molecular dynamics simulations of the heat capacity of Cu-Ag alloy. J Eng Thermophys (in Chinese), 2002, 23(3): 274-276

6 Daw M S, Basks M I. Embedded-atom method: Derivation and application to impurities, surface and other defects in metals. Phys Rev B, 1984, 29(12): 6443-6453 
7 Voter F, Chen S P. Accurate interatomic potential for Ni, Al, and $\mathrm{Ni}_{3} \mathrm{Al}$. Mater Res Soc Sympos Proc, 1987, 82: 175-180

8 Toxvaerd S, Prastgaard E. Molecular dynamics calculation of the liquid structure up to a solid surface. J Chem Phys, 1977, 67(11): 5291-5295

9 Hiwatari Y, Stoll E, Schneider T. Molecular-dynamics investigation of solid-liquid coexistence. J Chem Phys, 1978, 68(8): $3401-3404$

10 Mori A, Manabe R, Nishioka K. Construction and investigation of a hard-sphere crystal-melt interface by a molecular dynamics simulation. Phys Rev E, 1995, 51(5): R83831-R3833

11 Han X J. Molecular dynamics study of thermophysical properties and structure of undercooled metallic melts. Research Report for Post-doctor. Beijing: Tsinghua University, 2004

12 Morris J R, Song X. The melting lines of model systems calculated from coexistence simulations. J Chem Phys, 2002, 116(21): 9352-9358

13 Yoo S, Zeng X C, Morris J R. The melting lines of model silicon calculated from coexisting solid-liquid phases. J Chem Phys, 2004, 120(3): 1654-1656

14 Wang H L, Wang X X, Liang H G. Molecular dynamics simulation and analysis of bulk and surface melting processes for metal Cu. Acta Metallrugica Sinica (in Chinese), 2005, 41(6): 568-572

15 Qi L, Zhang H F, Hu Z Q. Molecular dynamic simulation of melting and solidification in binary liquid metal: Cu-Ag. Acta Metallrugica Sinica (in Chinese), 2004, 40(7): 736-740

16 Mohan P V, Suryanarayana S V, Satyanarayna K, et al. The high-temperature thermal expansion of $\mathrm{Ni}_{3} \mathrm{Al}$ measured by X-ray diffraction and dilation methods. J Phys Condens Matter, 1989, 1(32): 5357-5361

17 Feenken J W M, van der Veen J F. Observation of surface melting. Phys Rev Lett, 1985, 54(2): 134-137 